\title{
Caracterización molecular de Escherichia coli y Klebsiella pneumoniae productores de $\beta$-lactamasas de espectro extendido en hospitales de la Región Caribe, Colombia
}

\author{
Sandra L. Gaitán C., Paula A. Espinal M. y Grupo de Investigación en Resistencia Bacteriana, Región Caribe
}

\section{Molecular characterization of extended-spectrum $\beta$-lactamases-producing Escherichia coli and Klebsiella pneumoniae in hospitals of the Caribean Region, Colombia}

ESBL-producing in Enterobacteriaceae, is the main resistance mechanism to extended spectrum cephalosporin and monobactams. Seventy isolates collected of hospitals in four cities of the Colombian Caribean, were characterized to ESBL production and metalo- $\beta$-lactamases by microbiological test. The $\beta$-lactamases characterization were performed by IEF and RPC; genotyping by PFGE. Results evidenced the ESBL production at four cities with more frequency in Klebsiella pneumoniae isolated from UCI. The $\beta$-lactamases present in Escherichia coli and in $K$. pneumoniae contributed co-resistance to different antibiotic families. Enzymes were detected with resistance to cephalosporin and carbapenems, suggesting presence of carbapenemases. Polyclonal isolates noticed, neither demonstrated presence of endemic strains nor association with epidemic outbreak. It is evident the importance to combine clinical, microbiological and molecular information to surveillance the prevalence and evolution of these enzymes in these hospitals.

Key words: Escherichia coli, Klebsiella pneumoniae, $\beta$-lactamases, ESBL, Colombia.

Palabras clave: Escherichia coli, Klebsiella pneumoniae, $\beta$-lactamasas, BLEE, Colombia.

\section{Introducción}

$\mathrm{D}$ urante las últimas décadas, las $\beta$-lactamasas de espectro extendido (BLEE) del tipo TEM, SHV, OXA, y recientemente, CTX-M descritas en bacilos gramnegativos han emergido como un mecanismo significativo de resistencia ${ }^{1,2}$. Las BLEE son enzimas capaces de hidrolizar eficientemente cefalosporinas de espectro extendido (cefotaxima y ceftazidima, entre otras) y monobactámicos (aztreonam) por lo que se han asociado con fallas terapéuticas. Estas enzimas se han diseminado peligrosamente en amplias regiones geográficas ${ }^{3}$ y el éxito de esta diseminación se debe probablemente a que el gen de resistencia (bla $a_{\mathrm{TEM}}, b l a_{\mathrm{SHV}}$, entre otros) es frecuentemente transportado en plásmidos auto-transmisibles o móviles, capaces de diseminarse horizontalmente entre e intra especies ${ }^{4}$. Según el reporte del proyecto SENTRY, Klebsiella pneumoniae y Escherichia coli con fenotipo de BLEE fueron más frecuentes en América Latina, seguida por la región del pacífico occidental, Europa, E.U.A. y Canadá. En Colombia, según lo reportado por CIDEIM, se encontró entre 8 y $11 \%$ en E. coli y 20 a $30 \%$ en $K$. pneumoniae con fenotipos sugestivos de
BLEE $^{6}$ y se describió la primera BLEE tipo cefotaximasa (CTX-M-12) en el país?.

En el presente estudio se realizó la caracterización molecular de $\beta$-lactamasas en aislados clínicos de $E$. coli y K. pneumoniae recuperadas de seis instituciones hospitalarias en cuatro ciudades de la región Caribe, entre marzo de 2005 y agosto de 2006.

\section{Materiales y Métodos}

Aislados bacterianos y caracterización microbiológica. De 144 aislados consecutivos de E. coli y $K$. pneumoniae recuperados de pacientes hospitalizados en diferentes servicios, el análisis se llevó a cabo con 70 que fueron resistentes a alguna oximino-cefalosporina, recuperadas durante 18 meses en seis instituciones de tercer nivel de atención en salud de las ciudades de Barranquilla (19), Cartagena (16), Montería (29) y Sincelejo (6). Los microorganismos fueron identificados a nivel de especie por medio del sistema MicroScan (NC32, Dade Behring).

El tamizaje y la prueba confirmatoria para la producción de BLEE fueron realizadas por el método de difu-
Universidad del Sinú, Montería, Colombia

Facultad de Ciencias de la Salud Grupo de Enfermedades Tropicales y Resistencia Bacteriana

Recibido: 6 de febrero 2008 Aceptado: 14 de enero 2009

Los autores declaran que no existen conflictos de interés.

Este trabajo fue financiado por el Instituto Colombiano para el desarrollo de la Ciencia y Tecnología "Francisco José de Caldas" COLCIENCIAS (Código:1283-0416546) y por la Universidad del Sinú.

Correspondencia a: Paula A. Espinal Marín paulaespinal@unisinu.edu.co 
sión en disco (Oxoid) en agar Mueller Hinton, de acuerdo con las guías del Clinical and Laboratory Standards Institute $^{8}$ (CLSI, Enterobacteriaceae M2-Disk Diffusion Table 2A, 2006).

El tamizaje de productores de metalo- $\beta$-lactamasas $(\mathrm{M} \beta \mathrm{L})$, se llevó a cabo empleando la prueba de sinergia de doble disco: imipenem-EDTA $(10 \mu \mathrm{g} / 750 \mu \mathrm{g}) \mathrm{y}$ ceftazidima-EDTA $(30 \mu \mathrm{g} / 750 \mu \mathrm{g})^{9,10}$. Los microorganismos fueron inoculados sobre placas de agar Mueller Hinton según $\mathrm{CLSI}^{8}$ y se siguió el protocolo establecido por Hemalatha y cols ${ }^{11}$. Las zonas de inhibición de los discos de imipenem y ceftazidima, con y sin EDTA, fueron comparados después de 18 horas de incubación a $35^{\circ} \mathrm{C}$; la prueba de tamizaje fue considerada positiva si la diferencia en la lectura de halos de los discos solos y en combinación con EDTA era $\geq 7 \mathrm{~mm}$.

Las cepas ATCC 25922 de E. coli y ATCC 700603 de $K$. pneumoniae, fueron usadas como control negativo y positivo para la producción de BLEE, respectivamente. La cepa ATCC 27853 de Pseudomonas aeruginosa (susceptible a imipenem) fue usada como control negativo para la producción de M $\beta \mathrm{L}$.

\section{Caracterización de $\beta$-lactamasas}

Isoelectroenfoque. Se realizó la caracterización inicial de las $\beta$-lactamasas en todos los aislados seleccionados por iso-electoenfoque (IEF) de acuerdo al método de Mathew y cols ${ }^{12}$, con el modelo 111 Mini IEF Cell (Bio-Rad, Hercules, Calif.). Las preparaciones de $\beta$ lactamasas libres de células fueron obtenidas por cuatro sonicaciones, cada una durante $10 \mathrm{~s}$ con intermitente enfriamiento sobre hielo. Los sobrenadantes $(10 \mu \mathrm{l})$ de cada preparación fueron sometidos a IEF sobre geles de poliacrilamida (anfolitos con rango $\mathrm{pH} 3-9,5$ ) y separados a $100 \mathrm{~V}$ (15 $\mathrm{min}), 200 \mathrm{~V}$ (15 $\mathrm{min})$ y $450 \mathrm{~V}$ (60 $\mathrm{min}$ ) con el sistema Bio-Rad's PowerPac 3000. Después del IEF, la actividad de las $\beta$-lactamasas fue revelada con nitrocefina $250 \mu \mathrm{M}$ (Oxoid Ltd, Basingstoke, Hampshire, England). El punto isoeléctrico (pI) fue identificado por comparación con enzimas de referencia (TEM-1: 5,4, SHV-3: 7,0, SHV-2: 7,6, SHV-5: 8,2 y TLA-1: 9,0) corridos en carriles adyacentes a las muestras.
Reacción de polimerasa en cadena (RPC) para la detección de genes bla. Todas las cepas fueron evaluadas para la presencia de $b l a_{\mathrm{TEM}}, b l a_{\mathrm{SHV}}, b l a_{\mathrm{CTX}-\mathrm{M}}$ específicamente del grupo 1 y $b l a_{\mathrm{AmpC}}$ usando las condiciones de RPC descritas previamente ${ }^{1,13}$. Los iniciadores son descritos en la Tabla 1 . Los productos de RPC se resolvieron sobre geles de agarosa al $0,8 \%$, teñidos con bromuro de etidio y fotografiados con el sistema ChemiDoc XRS ${ }^{\mathrm{TM}}$ (Bio-Rad).

\section{Genotipificación}

El análisis por electroforesis en gel por campos pulsados (pulsed-field gel electrophoresis, PFGE) fue realizado siguiendo lo descrito por Miranda y cols ${ }^{14}$, con la ezima de restricción XbaI (Invitrogen) y el sistema CHEF DRII PFGE (Bio-Rad). Las comparaciones para $E$. coli y $K$. pneumoniae fueron hechas usando el coeficiente de Dice. Los dendrogramas fueron generados usando el método de UPGMA (unweighted pair group method using arithmetic averages). La relación de ADN fue calculada sobre el coeficiente de Dice y los aislados fueron considerados genéticamente relacionados si la correlación del coeficiente de Dice fue de $85 \%$ o mayor lo cual corresponde al criterio "posiblemente relacionado" (4-6 bandas diferentes) de Tenover y cols ${ }^{15}$.

\section{Resultados}

\section{Identificación y pruebas de susceptibilidad}

Distribución de los aislados clínicos. De 144 aislados de E. coli y $K$. pneumoniae recuperados dentro del período de estudio, 48,6\% de estos (70 aislados) fueron considerados resistentes a alguna oximinocefalosporina. De ellos, $39(55,7 \%)$ correspondieron a E. coli y $31(44,2 \%)$ a $K$. pneumoniae.

El servicio hospitalario del cual se obtuvo la mayoría de aislados fue la UCI, $(44,3 \%, 31 / 70)$, seguido de hospitalizados $(25,7 \%, 18 / 70)$ y medicina interna $(20 \%$, 14/70). En UCI, K. pneumoniae fue el microorganismo predominante $(74,2 \%, 23 / 31)$, mientras que en hospita-

\begin{tabular}{|c|c|c|c|c|}
\hline Gen & & & $\mathbf{T}^{\circ}$ de asociación & $\begin{array}{c}\text { Tamaño } \\
\text { producto (pb) }\end{array}$ \\
\hline$b^{b l a}{ }_{\text {TEM }}{ }^{1}$ & $5^{\prime}$-AAA CGC TGG TGA AAG TA 3' & 5'-AGC GAT CTG TCT AT 3' & $45^{\circ} \mathrm{C}$ & 239 \\
\hline$b / a_{\mathrm{SHV}}{ }^{1}$ & 5' - ATG CGT TAT ATT CGC CTG TG 3' & 5'-TGC TTT GTT ATT CGG GCC AA 3' & $60{ }^{\circ} \mathrm{C}$ & 241 \\
\hline 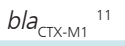 & $5^{\prime}-$ GAC GAT GTC ACT GGC TGA GC 3' & $5^{\prime}$-AGC CGC CGA CGC TAA TAC A 3' & $55^{\circ} \mathrm{C}$ & 499 \\
\hline Amp-C ${ }^{1}$ & 5'-ATC AAA ACT GGC AGC CG-3' & 5'-GAG CCC GTT TTA TGC ACC CA-3' & $60^{\circ} \mathrm{C}$ & 170 \\
\hline
\end{tabular}


lizados y medicina interna predominó E. coli en $33,4 \%$ $(13 / 39)$ y $30,1 \%(12 / 39)$, respectivamente.

El origen de las muestras a partir de las cuales se obtuvieron los aislados, fue: orina $(47,1 \%, 33 / 70)$, sangre $(17,2 \%, 12 / 70)$, secreción bronquial $(8,6 \%, 6 / 70)$ y secreción de tejidos blandos $(7,1 \%, 5 / 70)$. Escherichia coli predominó en muestras de orina $(81,3 \%, 27 / 33)$ y $K$. pneumoniae en sangre $(58,3 \%, 7 / 12)$ y secreción bronquial $(100 \%, 6 / 6)$.

\section{Susceptibilidad antimicrobiana y detección de cepas productoras de BLEE}

Los 70 aislados presentaron un perfil sugestivo de BLEE, mostrando resistencia a algunos de los antimicrobianos establecidos para el tamizaje. En las Figuras 1 y 2 se aprecia la frecuencia de resistencia intermedia y alta observada en $K$. pneumoniae y $E$. coli frente a diferentes grupos de antimicrobianos. La multi-resistencia observada incluye cefalosporinas de tercera generación, aminoglucósidos, quinolonas, cotrimoxazol y las combinaciones de $\beta$-lactámicos con inhibidores de $\beta$-lactamasas; en su mayoría, las resistencias fueron más frecuentes en $K$. pneumoniae que en E. coli.

No se observó resistencia a carbapenems en aislados de $E$. coli, mientras que en $K$. pneumoniae se observó $9,7 \%$ a imipenem y 6,5\% a meropenem. De tres aislados de K. pneumoniae, dos evidenciaron resistencia simultánea a imipenem y meropenem y uno solo a imipenem. La prueba de tamizaje para $\mathrm{M} \beta \mathrm{L}$ fue negativa, lo cual indicó que el mecanismo de resistencia no era la producción de estas enzimas y sugirió la presencia de otros mecanismos de resistencia como carbapenemasas clase $\mathrm{A}$, presencia de bombas de eflujo o modificación de las porinas de membrana (en estudio).

Se confirmó la presencia de BLEE en 28/70 aislados, 12 de E. coli y 16 de $K$. pneumoniae por medio de la prueba confirmatoria con ácido clavulánico. Los discos de cefotaxima y cefotaxima/ác. clavulánico confirmaron la presencia de BLEE en 10 de 12 aislados de $E$. coli y 15 de 16 cepas de K. pneumoniae. Los discos de ceftazidima y ceftazidima/ác. clavulánico demostraron menor capacidad para detectar BLEE, 11 de 12 aislados de E. coli y 12 de 16 cepas de $K$. pneumoniae.

De los 70 aislados resistentes a alguna cefalosporina, 42 no fueron inhibidos por ácido clavulánico; sin embargo, fueron caracterizados por IEF y RPC teniendo en cuenta su perfil de susceptibilidad.

\section{Caracterización de las $\beta$-lactamasas}

El análisis de IEF demostró la presencia de varias $\beta$ lactamasas en los 70 aislados estudiados que fueron resistentes a alguna cefalosporina.

El número de $\beta$-lactamasas producidas por cada uno de los aislados fue variable, encontrándose en

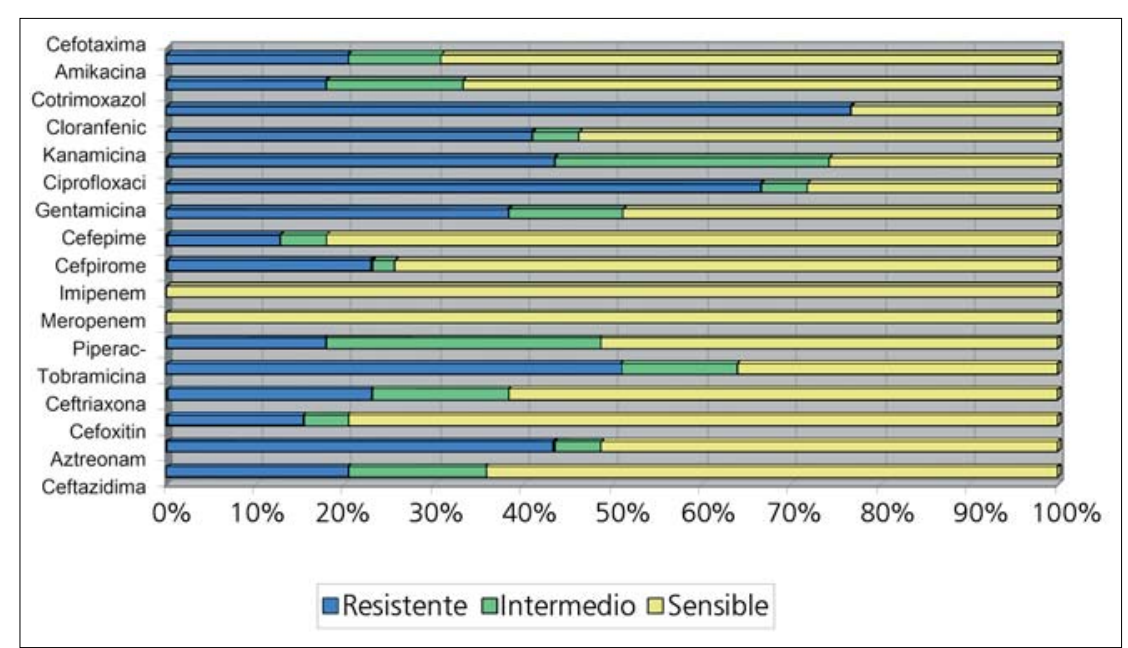

Figura 1. Susceptibilidad antimicrobiana en aislados con tamizaje de BLEE positivo en Escherichia coli, $\mathrm{n}=39$.

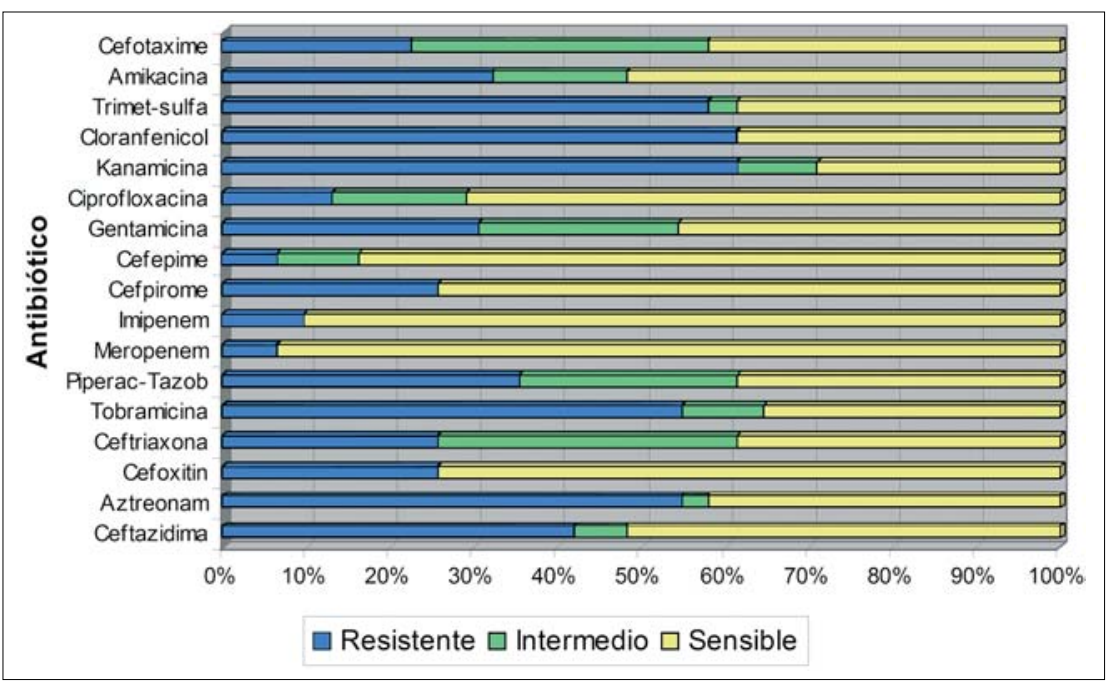

Figura 2. Susceptibilidad antimicrobiana en aislados con tamizaje de BLEE positivo en Klebsiella pneumoniae, $\mathrm{n}=31$.

algunos casos la producción simultánea de dos o más enzimas, como se observa en los datos obtenidos: producción de una enzima (61,4\%; 43 aislados), dos enzimas ( $37,1 \% ; 26$ aislados) y tres $(1,4 \% ; 1$ aislado).

Dentro del grupo de aislados que expresaron sólo un pI, el más común fue 5,4 , presente en 14 aislados de K. pneumoniae y 24 de E. coli.

De los 28 aislados confirmados como productores de BLEE, diez tuvieron un pI de 5,4; dos aislados de 5,8 y uno de 7,0. Los 15 aislados restantes tuvieron producción simultánea de pI diferentes.

El pI de los 42 aislados con prueba confirmatoria de BLEE negativa es detallado en la Tabla 2. 


\begin{tabular}{|c|c|c|}
\hline pl & $\begin{array}{l}\mathrm{N}^{\circ} \text { de aislados de } \\
\text { Escherichia coli }\end{array}$ & $\begin{array}{l}\mathbf{N}^{\circ} \text { de aislados de } \\
\text { Klebsiella pneumoniae }\end{array}$ \\
\hline 5,4 & 21 & 9 \\
\hline 5,2 y 8,9 & 4 & 1 \\
\hline 5,3 y 9,2 & 2 & 3 \\
\hline 5,4 y 7,0 & 0 & 2 \\
\hline
\end{tabular}

Se amplificó cada aislado que fue resistente a alguna cefalosporina con iniciadores específicos para los genes $b l a_{\mathrm{TEM}}, b l a_{\mathrm{SHV}}, b l a_{\mathrm{CTX}-\mathrm{M}}$ del grupo 1 y $b l a_{\mathrm{AmpC}}$.

Dentro de los aislados estudiados, 87,1\% (61/70) amplificaron para el gen $b l a_{\mathrm{TEM}} ; 41,4 \%(29 / 70)$ para $b l a_{\text {Ampc }} ; 38,6 \%(27 / 70)$ para el gen $b l a_{\text {SHV }}$ y $32,9 \%(23 / 70)$ para $b l a_{\text {СТХ-M1 }}$ (Tabla 3 ).

Se presentó amplificación simultánea de más de un gen en 39 de los 70 aislados $(55,7 \%)$. En cuatro aislados con pI evidenciado por IEF no se logró un producto de amplificación con alguno de los iniciadores específicos usados.

En los 28 aislados productores de BLEE se encontró que el gen amplificado en $89,3 \%(25 / 28)$ fue $b l a_{\mathrm{TEM}}$; $85,7 \%(24 / 28)$ del gen $b l a_{\mathrm{SHV}} ; 64,3 \%(18 / 28)$ del gen $b l a_{\text {AmpC }}$ y $53,6 \%(15 / 28)$ para $b l a_{\text {СтХ-м }}$ del grupo 1. Más de un gen fue amplificado en $85,7 \%(24 / 28)$ de los casos (Tabla 3 ).

De los 42 aislados de $K$. pneumoniae y E. coli resistentes a alguna cefalosporina pero que tuvieron una prueba confirmatoria negativa para BLEE, más de un gen fue amplificado en 35,7 \% (15) de los casos. Los aislados de $K$. pneumoniae resistentes a carbapenems (imipenem y meropenem) evidenciaron la producción por IEF y RPC de la enzima TEM (dos aislados) y un tercer caso, la co-existencia de TEM, SHV, CTX-M1 y AmpC.

La presencia de dos o tres $\beta$-lactamasas involucradas en resistencia a oximino-cefalosporinas, fue observada en 43,6\% (17/39) de los aislados de E. coli y en $70,1 \%(22 / 31)$ de los aislados de K. pneumoniae.

En general, la amplificación sugirió la presencia si- multánea de genes $b l a_{\mathrm{TEM}}-b l a_{\mathrm{AmpC}}, \quad b l a_{\mathrm{SHV}}-b l a_{\mathrm{AmpC}} \mathrm{y}$ $b l a_{\text {СтХ-м }}$ del grupo 1- $b l a_{\mathrm{AmpC}}$ que fue confirmada en $38,6 \%$ (27/70 aislados), de los cuales 18\% (7/39 aislados) correspondieron a $E$. coli y $64,5 \%$ (20/31 aislados) a $K$. pneumoniae.

De un total de 70 aislados, se evidenció en 44 un pI que sugirió la presencia $\beta$-lactamasas tipo TEM (pI 5,3-5,6). Sin embargo, por RPC se confirmó amplificación con los iniciadores para el gen $b l a_{\mathrm{TEM}}$ en 61 cepas. De estos aislados, se amplificó simultáneamente los genes $b l a_{\text {TEM }}$ y $b l a_{\text {SHV }}$ en $26, b l a_{\text {TEM }}$ y $b l a_{\text {СTХ-M }}$ del grupo 1 en 23 y $b l a_{\text {TEM }}$ y $b l a_{\text {AmpC }}$ en 25 aislados.

Se observó amplificación de bandas con tamaños esperados para los genes de BLEE, predominantemente en $E$. coli de $b l a_{\text {SHV }}$ y $b l a_{\text {СтХ-M }}$ del grupo 1 y para $K$. pneumoniae de $b l a_{\mathrm{AmpC}}$ y $b l a_{\mathrm{SHV}}$. Cuando se relacionó la amplificación de estas bandas con el perfil de susceptibilidad se encontraron las siguientes asociaciones:

- La amplificación simultánea de bandas posiblemente de los genes $b l a_{\mathrm{TEM}}$ y $b l a_{\mathrm{SHV}}$ en $E$. coli, se observó que los mayores porcentajes de resistencia estuvieron asociados a ceftazidima, aztreonam, ceftriaxona, cefotaxima, cefepime, amikacina y ciprofloxacina.

- La coexistencia de bandas posiblemente de los genes $b l a_{\text {TEM }}$ y $b l a_{\text {СTX-M }}$ del grupo 1 en E. coli, mostró mayor resistencia principalmente a cefoxitina, piperacilina/tazobactam, cefotaxima, cotrimoxazol, gentamicina, kanamicina y amikacina.

- Cuando se presentó la amplificación simultánea de bandas posiblemente de los genes $b l a_{\mathrm{TEM}}$ y $b l a_{\mathrm{AmpC}}$ para $K$. pneumoniae, se presentó resistencia a todos los antimicrobianos valorados, excepto ceftriaxona que mostró un mayor porcentaje para la coexistencia de los genes $b l a_{\mathrm{TEM}}$ y $b l a_{\mathrm{SHV}}$, situación en la que se observó porcentajes de resistencia iguales a los asociados con la presencia de $b l a_{\text {TEM }}$ y $b l a_{\text {AmpC }}$ en ceftazidima, cefoxitina, carbapenems y cefepime.

\section{Tipificación por electroforesis en gel de campo pulsado}

Se realizó la genotipificación de la totalidad de los aislados (n: 144) con el fin de establecer cualquier

Tabla 3. Presencia de genes bla en Escherichia coli y Klebsiella pneumoniae

\begin{tabular}{|c|c|c|c|c|c|c|c|c|}
\hline \multirow{2}{*}{$\begin{array}{l}\text { Resultado de la prueba } \\
\text { confirmatoria de } \\
\text { producción de BLEE }\end{array}$} & \multicolumn{4}{|c|}{$\begin{array}{c}\text { Presencia del gen bla en } \\
\text { Escherichia coli }\end{array}$} & \multicolumn{4}{|c|}{$\begin{array}{l}\text { Presencia del gen bla en } \\
\text { Klebsiella pneumoniae }\end{array}$} \\
\hline & $\mathbf{b l a}_{\text {стX-M1 }}$ & bla $_{\text {TEM }}$ & bla $_{\text {SHV }}$ & bla $_{\text {AmpC }}$ & $\mathbf{b l a}_{\text {crX-M1 }}$ & bla $_{\text {TEM }}$ & bla $_{\text {SHV }}$ & bla $_{\text {AmpC }}$ \\
\hline Positiva & 7 & 11 & 10 & 7 & 8 & 14 & 14 & 11 \\
\hline Negativa & 6 & 23 & 0 & 0 & 2 & 13 & 3 & 10 \\
\hline Total & 13 & 34 & 10 & 7 & 10 & 27 & 17 & 21 \\
\hline
\end{tabular}


Tabla 4. Descripción de los clones obtenidos a partir de los 144 aislados de Klebsiella pneumoniae y Escherichia coli

\begin{tabular}{|c|c|c|c|c|c|c|}
\hline Clon & $\begin{array}{c}\text { Aislado } \\
\text { Klebsiella pneumoniae }\end{array}$ & Fecha de recolección & Servicio & Fuente & pl* & Genes bla \\
\hline \multirow[t]{2}{*}{$A$} & CC34 & Diciembre 2005 & $\mathrm{UCl}$ & Sec. bronquial & 5.2 y 9.2 & TEM y AmpC \\
\hline & $\mathrm{CC} 42$ & Enero 2006 & Hospitalizados & Sangre & 5.4 y 9.2 & TEM \\
\hline \multirow[t]{2}{*}{ B } & HSJ13 & Marzo 2005 & Urgencias & Sangre & 5.4 & TEM, AmpC, SHV y CTX-M1 \\
\hline & HSJ14 & Mayo 2005 & UCI & Sec. vesical & 5.4 & TEM, AmpC, SHV y CTX-M1 \\
\hline Clon & $\begin{array}{c}\text { Aislado } \\
\text { Escherichia coli }\end{array}$ & Fecha de recolección & Servicio & Fuente & $\mathbf{p} \mathbf{l}^{*}$ & Genes bla \\
\hline \multirow[t]{2}{*}{$A$} & HSJ3 & Febrero 2005 & Hospitalizados & Orina & 5.2 y 8.9 & TEM \\
\hline & HSJ6 & Marzo 2005 & Cirugía & Sec. tejido blando & 5.0 y 8.8 & TEM \\
\hline \multirow[t]{2}{*}{ B } & $\mathrm{HI3}$ & Marzo 2005 & Cirugía & Orina & 5.4 y 7.6 & TEM, SHV, CTX-M1 \\
\hline & $\mathrm{HB} 1$ & Diciembre 2005 & Cirugía & Líq. peritoneal & 5.4 & TEM \\
\hline
\end{tabular}

relación clonal. El análisis de los perfiles electroforéticos mostró, en promedio, la presencia de 27 a 30 bandas entre 50 y $1050 \mathrm{~Kb}$. Con los perfiles electroforéticos obtenidos se generaron matrices de presencia (1) y ausencia (0), que fueron analizados con el software NTSYS PC 2.0. Para los dendrogramas obtenidos se estableció como porcentaje de similaridad el $85 \%$.

El análisis de PFGE de los 144 aislados, indicó que en las 51 cepas de $K$. pneumoniae se encontraron dos clones. Un clon A dos aislados con una similaridad del $87 \%$ (CC34 y CC42) y el clon B con dos cepas idénticas (HSJ13 y HSJ14) (Tabla 4).

Para los 93 aislados de E. coli, se observaron dos clones. El clon A incluyó dos aislados, HSJ3 y HSJ6 con una similaridad de $92 \%$. El clon B incluyó los aislados HI3 y HB1 con $83 \%$ de similaridad, recuperados de fuente e instituciones diferentes. Un aspecto en común fue el servicio (cirugía) en el cual se recuperó el microorganismo (Tabla 4).

Los aislados restantes para los dos microorganismos constituyeron un grupo heterogéneo con perfiles electroforéticos muy diferentes.

En general, los resultados obtenidos por tipificación por PFGE no evidenciaron relación clonal entre los aislados, lo cual fue consistente con la ausencia de un perfil característico en las pruebas de susceptibilidad.

\section{Discusión}

Los datos sobre la actividad de varios agentes antimicrobianos contra aislados de $E$. coli y $K$. pneumoniae productores de BLEE, muestran que la combinación $\beta$-lactámico más inhibidores de $\beta$ - lactamasas (IBL), carbapenems, aminoglucósidos y fluoroquinolonas son medicamentos potencialmente activos contra microorganismos productores de BLEE $^{16,17}$. Los resultados de los agentes antimicrobianos probados en este estudio demostraron que solamente los carbapenems tuvieron $100 \%$ de actividad contra aislados de E. coli, seguidos por amikacina, cefoxitina y piperacilina/tazobactam. Para K. pneumoniae hubo buena actividad con cefoxitina, ciprofloxacina, amikacina y carbapenems. Sin embargo, se encontró resistencia, inclusive a imipenem y meropenem, lo cual demuestra, que en las instituciones incluidas en el estudio sólo algunas de estas alternativas terapéuticas continúan siendo eficaces, y que ya se encuentran presentes aislados con varias enzimas y mecanismos co-existentes que confieren multi-resistencia, situación que ha sido reportado en otras regiones geográficas ${ }^{18,19}$.

Aunque la resistencia a carbapenems en $K$. pneumoniae se detecta con baja frecuencia, en el presente estudio se encontraron aislados resistentes que fueron negativos a la prueba de tamizaje usando EDTA, lo que descartó la producción de M $\mathrm{ML}$ y sugirió como posible mecanismo de resistencia la producción de carbapenemasas, enzimas reportadas recientemente en Colombia $^{20}$. Debido a que la incidencia de estas enzimas en enterobacterias es muy baja y produce una resistencia de alto nivel, no sólo a imipenem, sino también al resto de $\beta$-lactámicos, actualmente se realizan estudios para su confirmación.

La resistencia asociada a aminoglucósidos fue observada en cepas de ambas especies, lo cual es consistente con lo reportado en la literatura científica donde la producción de la enzima tipo CTX-M en aislados de 
E. coli y Klebsiella sp se ha asociado con resistencia a otros antimicrobianos, tales como ciprofloxacina y aminoglucósidos ${ }^{21}$.

La resistencia a fluoroquinolonas se observó principalmente en E. coli y, debido al alto nivel de resistencia observado $(72 \%)$, es posible que varios mecanismos simultáneamente se encuentren asociados, entre ellos la reducción en la permeabilidad, bombas de eflujo y mutaciones en las topo-isomerasas. Aunque en $K$. pneumoniae la sensibilidad a este antimicrobiano fue buena $(71 \%)$, es importante informar el porcentaje de aislados con resistencia intermedia, $16 \%$, ya que esta situación aumenta la probabilidad de adquirir resistencia a fluoroquinolonas y el consiguiente riesgo de fracaso terapéutico ${ }^{22}$.

La prueba confirmatoria de productores de BLEE, permitió detectar 28 cepas y aunque la prueba fue negativa para los 42 restantes, se demostró por RPC la amplificación de bandas que sugirieron la presencia de genes bla. Esto puede ser explicado por la presencia de enzimas contra inhibidores de $\beta$-lactámicos, hiperproducción de enzimas, asociación con enzimas tipo AmpC o la presencia de otros tipos de mecanismos de resistencia. Al correlacionar esta información con los perfiles de susceptibilidad no se encontró concordancia, análisis similares han sido reportados por Tenover et $\mathrm{al}^{23}$.

Los resultados de esta investigación revelan que la presencia de BLEE posiblemente de tipo TEM, CTX-M del grupo 1 y SHV, fueron encontradas en todos los hospitales participantes y en varios aislados se demostró la amplificación de bandas asociadas a más de un posible gen bla (TEM, CTX-M del grupo 1, SHV y/ o AmpC) en una misma cepa, lo cual es coherente con lo reportado por otros investigadore ${ }^{24-27}$. La distribución de las $\beta$-lactamasas confirmó variabilidad en la epidemiología de estos determinantes de resistencia y evidenció la necesidad de monitoreo constante.

Aunque la mayoría de las BLEE, se sugiere, son del tipo TEM y SHV, las CTX-M se han reportado de manera incrementada en bacilos gramnegativos ${ }^{2}$. En Colombia las enzimas CTX-M, especialmente del grupo 1 , se están reportando con mayor frecuencia ${ }^{28-30}$, lo cual fue concordante con los resultados obtenidos en este estudio.

Se esperaba que la resistencia a ceftazidima fuera, en general, más baja que para cefotaxima, especialmente en aislados sospechosos de ser productores de CTX-M1; sin embargo, en nueve no se observó inhibición por ácido clavulánico. Lo anterior demuestra que el fenotipo clásico de resistencia conferido por la BLEE tipo CTX-M no es universal y que la producción simultánea de $\beta$-lactamasas adicionales en los 23 aislados constituye un factor que posiblemente haya alte- rado la especificidad de sustrato de CTX-M, enmascarando su presencia ${ }^{17,31}$.

Siete aislados (5 de E. coli y 2 de $K$. pneumoniae) no fueron detectados inicialmente como productores de BLEE, ya que fueron resistentes a clavulanato y a piperacilina/tazobactam y sólo por RPC se evidenciaron bandas asociadas con el gen $b l a_{\mathrm{TEM}}$. Un resultado similar fue reportado por Robin et $\mathrm{al}^{32}$, donde explica que el uso de cefalosporinas de espectro extendido ha conducido a la selección de BLEE, detectándose enzimas que combinan BLEE y mutaciones ITR (inhibitor-resistant enzymes) que ocurren en la región de los genes $b l a_{T E M}$ responsables de la producción de TEM-1y TEM-2, confiriendo resistencia cruzada a las combinaciones de amoxicilina/ác. clavulánico, ampicilina/sulbactam, amoxicilina/tazobactam y piperacilina/ tazobactam. Se han identificado en E. coli, $K$. pneumoniae, Proteus mirabilis y Enterobacter aerogenes. Es posible que este fenotipo encontrado en los aislados analizados corresponda a la nueva enzima por lo que se hace necesario continuar su estudio para la confirmación de esta hipótesis.

Para las cepas en las cuales se evidenció pI, pero no se logró amplificación con los iniciadores para los genes bla, se ampliará el estudio para la detección de otros tipos de $\beta$-lactamasas.

La relación clonal establecida por PFGE de este estudio, evidenció una gran variabilidad genética dentro de los aislados de E. coli y $K$. pneumoniae, lo cual sugiere la aparición de cepas resistentes específicas más que la circulación y transmisión de un aislado habitual entre pacientes. La policlonalidad observada en los aislados de cada especie sugiere que durante el tiempo del estudio no se demostró la presencia de cepas endémicas en las instituciones ni tampoco la asociación con brotes epidémicos. Sólo dos cepas productoras de BLEE de dos instituciones diferentes, demostraron tener similaridad por PFGE, situación que sugiere la transmisión cruzada entre salas de cirugía de las dos instituciones de la misma ciudad.

En conclusión, el aislado de microorganismos productores de BLEE es un problema presente en las cuatro ciudades de la región Caribe, que tiene mayor frecuencia, en términos absolutos, en $K$. pneumoniae, y que más de la mitad de éstos se aíslan en UCI. Se pudo determinar que los resultados obtenidos por RPC concuerdan con la posible presencia de cuatro tipos de $\beta$-lactamasas en los dos microorganismos, siendo en E. coli predominantes las asociadas a SHV y CTX- M1 y para $K$. pneumoniae las enzimas AmpC y $\mathrm{SHV}$, lo cual es concordante con lo reportado en otros estudios multicéntricos en los que se ha determinado que la enzima tipo CTX-M1 es más frecuente en $E$. coli у CTX-M9 en $K$. pneumoniae ${ }^{21,33}$. Debido a que $K$. 
pneumoniae no contiene genes constitutivos de AmpC, como sí sucede con E. coli, la presencia de bandas asociadas al gen $b l a_{\mathrm{AmpC}}$, evidencia la posibilidad del movimiento de estos genes sobre plásmidos como mecanismo de diseminación entre bacilos gramnegativos $^{34}$.

Este estudio multicéntrico es uno de los primeros en aportar información sobre la prevalencia y distribución de aislados E. coli y K. pneumoniae con fenotipo productor de BLEE, posibles tipos de enzimas producidas, relación clonal y susceptibilidad a medicamentos potencialmente activos dentro de algunas instituciones hospitalarias de la región Caribe Colombiana (2005-2006). Esta información contribuye al conocimiento de la epidemiología de la resistencia en cada institución, permite establecer la relación entre el uso de antimicrobianos y la resistencia bacteriana y de esta forma la implementación de recomendaciones para el manejo de antimicrobianos, precauciones de contacto y vigilancia activa de áreas de alto riesgo.

Agradecimientos. Al Instituto Colombiano para el desarrollo de la Ciencia y Tecnología "Francisco José de Caldas" COLCIENCIAS Proyecto código: 12830416546 Convenio: 471 y la Universidad del Sinú por la financiación del estudio, a Miguel Díaz por el apoyo técnico brindado y a Jesús Silva (INS, México) por la donación de cepas de referencia. De igual manera, al personal médico de las instituciones hospitalarias participantes por su valiosa colaboración: Hospital San Jerónimo: Llorente Genes y M. Helena
Díaz; Clínica de la Costa: Johana Soto; Clínica de la Asunción: Jefe Sandra Puello; Clínica de la Sabana: Wilmer Villamil e Ingrid Painchault; Clínica Santa María: Wilmer Villamil; Hospital Bocagrande: Wilfrido Coronel; Jefe Nasly Ortega y Julia Pimienta; Hospital Infantil: Inés Díaz y Hernando Pinzón.

\section{Resumen}

La producción de BLEE en Enterobacteriaceae, es el principal mecanismo de resistencia a cefalosporinas de espectro extendido y monobactámicos. Setenta aislados recuperados de hospitales de cuatro ciudades del Caribe colombiano, fueron caracterizados microbiológicamente para la producción de BLEE y metalo$\beta$-lactamasas. La caracterización de las $\beta$-lactamasas se realizó por IEF y RPC; la genotipificación por PFGE. Los resultados evidenciaron la producción de BLEE en las cuatro ciudades con mayor frecuencia en Klebsiella pneumoniae aislada de UCI. Las $\beta$ lactamasas presentes en Escherichia coli y en $K$. pneumoniae aportaron co-resistencia a diferentes familias de antimicrobianos. Se detectaron enzimas con resistencia a cefalosporinas y carbapenems, sugiriendo presencia de carbapenemasas. La policlonalidad observada no demostró presencia de cepas endémicas ni asociación con brotes epidémicos. Se evidencia la importancia de combinar datos clínicos, microbiológicos y moleculares para vigilar la prevalencia y evolución de $\beta$-lactamasas en estos hospitales.

\section{Referencias}

1.- Paterson D, Hujer K, Hujer A, Yeiser B, Bonomo M, Rice L, et al. Extendedspectrum $\beta$-lactamases in Klebsiella pneumoniae bloodstream isolates from seven countries: Dominance and widespread prevalence of SHV- and CTX-M-type $\beta$-lactamases. Antimicrob Agents Chemother 2003; 47 (11): 3554-60.

2.- Kim J, Lim Y, Jeong Y, Seol S. Ocurrence of CTX-M-3, CTX-M-15, CTX-M-14 and CTX-M-9 extended-spectrum $\beta$-lactamases in Enterobacteriaceae Clinical isolates in Korea. Antimicrob Agents Chemother 2005; 49 (4): 1572-5.

3.- Bradford P. Extended spectrum $\beta$-lactamases in the $21^{\text {st }}$ century: characterization, epidemiology, and detection of this important resistance in vitro. Clin Microbiol Rev 2001; 14: 933-51.

4.- Schwaber M J, Navon-Venezia S, Schwartz D, Carmeli Y. High levels of antimicrobial coresistance among extended spectrum $\beta$-lactamase producing Enterobacteriaceae. Antimicrob Agents Chemother 2005; 49: 2137-9.

5.- Gales C, Jones R, Gordon K, Sader H, Wilke W, Beach M. Activity and spectrum of antimicrobial agents tested again urinary tract infection pathogens in hospitalized patients in Latin America: report from the second year of the SENTRY antimicrobial surveillance program. J Antimicrob Chemother 1998; 45: 295-303.

6.- Villegas M V, Correa A, Pérez F, Miranda M C, Zuluaga T, Quinn J P, et al. Prevalence and characterization of extended-spectrum beta-lactamases in Klebsiella pneumoniae and Escherichia coli isolates from Colombian hospitals. Diagn Microbiol Infect Dis 2004; 49: 217-22.

7.- Villegas M V, Correa A, Pérez F, Zuluaga T, Radice M, Gutkind G, et al. CTX-M-12 betalactamase in a Klebsiella pneumoniae clinical isolate in Colombia. Antimicrob Agents Chemother 2004; 48: 629-31.
8.- Clinical and Laboratory Standards Institute. Performance standards for antimicrobial susceptibility testing. 2006; $16^{\text {th }}$ informational supplement.

9.- Yong D, Lee K, Yum J H, Shin H B, Rossolini G M, Chong Y. Imipenem-EDTA disk method for differentiation of metallo$\beta$-lactamase-producing clinical isolates of Pseudomonas spp. and Acinetobacter spp. J Clin Microbiol 2002; 40: 3798-801.

10.- Lee K, Lim Y S, Yong D, Yum J H, Chong Y. Evaluation of the Hodge test and the imipenem-EDTA double-disk synergy test for differentiating metallo- $\beta$-lactamase producing isolates of Pseudomonas spp. and Acinetobacter spp. J Clin Microbiol 2003; 41: 4623-9.

11.- Hemalatha V, Sekar U, Kamat V. Detection of metallo-betalactamase producing Pseudomonas aeruginosa in hospitalized Indian patients. J Med Res 2005; 122: 148-52.

12.- Mathew M, Harris A, Marshall M, Ross G. The use of analytical isoelectric focusing for 
detection and identification of $\beta$-lactamases. J General Microbiol 1975; 88: 169-78.

13.- Pitout J, Hossain A, Hanson N. Phenotypic and molecular detection of CTX-M- $\beta$ lactamases produced by Escherichia coli and Klebsiella spp. J Clin Microbiol 2004; 42: 5715-21.

14.- Miranda G, Kelly C, Solorzano F, Leanos B, Coria R, Paterson J. Use of pulsed-field gel electrophoresis typing to study an outbreak of infection due to Serratia marcescens in a neonatal intensive care unit. J Clin Microbiol. 1996; 34 (12): 3138-41.

15.- Tenover F, Arbeit R, Goering R, Mickelsen P, Murray B, Persing D, et al. Interpreting chromosomal DNA restriction patterns produced by pulsed field gel electrophoresis: Criteria for bacterial strain typing. J Clin Microbiol 1995; 33 (9): 2233-9.

16.- Luzzaro F, Mezzatesta M, Mugnaioli C, Perilli M, Stefani S, Amicosante G, et al. Trends in production of extended-spectrum $\beta$-lactamases among enterobacteria of medical interest: Report of the Second Italian Nationwide Survey. J Clin Microbiol 2006; 44 (5): 1659-64.

17.- Edelstein M, Pimkin M, Palagin I, Edelstein I, Stratchounski L. Prevalence and molecular epidemiology of CTX-M extended-spectrum $\beta$-lactamase-producing Escherichia coli and Klebsiella pneumoniae in Russian hospitals. Antimicrob Agents Chemother 2003; 47 (12): 3724-52.

18.- Giamarellou H. Multidrug resistance in Gram-negative bacteria that produce extended-spectrum $\beta$-lactamases (ESBLs). Clin Microbiol Infect 2005; 11 (Suppl 4): 1-16.

19.- Schwaber M J, Navon-Venezia S, Schwartz D, Carmeli Y. High levels of antimicrobial coresistance among extendedspectrum- $\beta$-lactamase producing Enterobacteriaceae. Antimicrob Agents Chemother 2005; 49: 2137-9.

20.- Villegas M, Lolans K, Correa A, Suárez C, Quinn J. Colombian Nosocomial Bacterial Resistance Study Group. First detection of KPC carbapenemases in Klebsiella isolates from South America. Program and Abstracts of $45^{\text {th }}$ Interscience Conference on Antimicrob Agents and Chemotherapy (ICAAC). American Society for
Microbiology Washington 2005; Abstract LB2-20.

21.- Eisner A, Fagan E, Feierl G, Kessler H, Marth E, Livermore D, Woodford N. Emergence of Enterobacteriaceae isolates producing CTX-M extended-spectrum $\beta$-lactamase in Austria. Antimicrob Agents Chemother 2006; 50 (2): 785-7.

22.- Cirz R, Romesberg F. Induction and inhibition of ciprofloxacin resistanceconferring mutations in hypermutator bacteria. Antimicrob Agents Chemother 2006; 50 (1): 220-5.

23.- Tenover F, Raney P, Williams $P$, Rasheed K, Biddle J, Oliver A, et al. Evaluation of the NCCLS extendedspectrum $\beta$-lactamase confirmation methods for Escherichiria coli with isolates collected during Project ICARE. J Clin Microbiol 2003; 41 (7): 3142-6.

24.- Spanu T, Luzzaro F, Perilli M, Amicosante G, Toniolo A, Fadda G, et al. Occurrence of extended-spectrum $\beta$-lactamases in members of the family Enterobacteriaceae in Italy: implications for resistance to $\beta$-lactams and other antimicrobial drugs. Antimicrob Agents Chemother 2002; 46: 196-202.

25.- Hernández J, Martínez L, Canton R, Coque T, Pascual A. Diversity of extendedspectrum beta-lactamases (ESBL) among E. coli and $K$. pneumoniae in a Nationwide Study in Spain. Program and Abstracts of $43^{\text {th }}$ Interscience Conference on Antimicrobial Agents and Chemotherapy (ICAAC) Chicago 2003; abstr C2-57.

26.- Leverstein V, Paauw A, Box A, Fluit A, Vehoef J. A Wide variety of (extendedspectrum) beta-lactamases detected in an outbreak of multiresistant Enterobacteriaceae in the Netherlands, including ACC-1, CTX-M-9, and novel TEM- and LEN-type beta-lactamases. Program and Abstracts of $43^{\text {th }}$ Interscience Conference on Antimicrobial Agents and Chemotherapy (ICAAC) Chicago 2003; abstr C2-49.

27.- Smith E, Geun S, Thomson K, Larone D, Hanson N. Letters to Editor. Klebsiella pneumoniae isolate producing at least eight different $\beta$-lactamases, including $\mathrm{AmpC}$ and
KPC $\beta$-lactamases. Antimicrob Agents Chemother 2007; 51 (2): 800-1.

28.- Espinal P, Garza U, Fernández A, Martínez P, Reyna F, Mattar S, et al. Extended spectrum $\beta$-lactamases (ESBL) identified in multiresistant Enterobacteria in Colombia hospitals, including CTX-M-12, SHV-2a, SHV-5, SHV-12 and novel SHV-86 $\beta$-lactamases. Program and Abstracts of $46^{\text {th }}$ Interscience Conference on Antimicrobial Agents and Chemotherapy (ICAAC), San Francisco. 2006. Abstr C2-98.

29.- Mantilla J, Reguero M, González E, García I, Leal A, Espinal P, et al. Caracterización molecular de un brote por Klebsiella pneumoniae productora de CTX-M-12 en la unidad de cuidado intensivo neonatal de un hospital colombiano. Biomédica 2006; 26 : 408-14.

30.- Villegas M, Correa A, Pérez F, Zuluaga T, Radice M, Gutkind G, et al. The Colombian Nosocomial Resistance Study Group. CTX-M-12 $\beta$-lactamase in a Klebsiella pneumoniae clinical isolate in Colombia. Antimicrob Agents Chemother 2004; 48: 629-31.

31.- Mugnaioli C, Luzzaro F, De Luca F, Brigante G, Perilli M, Amicosante G, et al. CTX-M-type extended-spectrum $\beta$-lactamases in Italy: Molecular epidemiology of an emerging countrywide problem. Antimicrob Agents Chemother 2006; 50 (6): 2700-6

32.- Robin F, Delmas J, Archambaud M, Schweitzer C, Chanal C, Bonnet R. CMT-type $\beta$-lactamase TEM-125, an emerging problem for extended-spectrum $\beta$-lactamase detection. Antimicrob Agents Chemother 2006; 50 (7): 2403-8.

33.- Yan J, Hsueh P, Lu J, Chang F, Shyr J, Wan $J$, et al. Extended-spectrum $\beta$-lactamases and plasmid-mediated AmpC enzymes among clinical isolates of Escherichia coli and Klebsiella pneumoniae from seven medical centers in Taiwan. Antimicrob Agents Chemother 2006; 50 (6): 1861-4

34.- Schmidtke A, Hanson N. Model system to evaluate the effect of ampD mutations on AmpC-mediated $\beta$-lactam resistance. Antimicrob Agents Chemother 2006; 50 (6): 2030-7. 